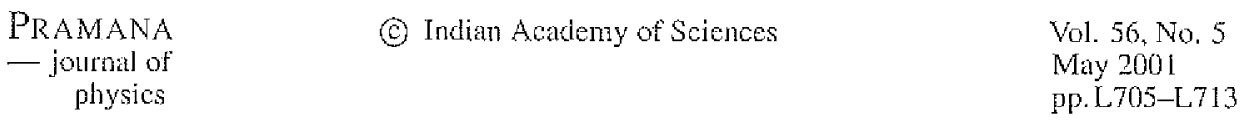

\title{
Quantum entanglement in the NMR implementation of the Deutsch-Jozsa algorithm
}

\author{
ARVIND, KAVITA DORAI* and ANIL. KUMAR' \\ Department of Physics, Guru Nanak Dev University, Amritsar 143005 , India \\ "Institute for Orgunic Chenistry. University of Frankfurt, D-60439 Frankfurt am Main, Germany \\ Sophisticated Instruments Facility, Indian Institute of Science, Bangalore 560 012, India \\ Email: arvind@physics.iisc.emet.in; kavita@physics.isc.emet.in; anilnmr@physics.isc.emet.in \\ MS received 1 February 2001; revised 13 April 2001 \\ Abstract. A scheme to execute an $n$-bit Deutsch-Jozsa (DJ) algorithm using $n$ qubits has been \\ implemented for up to three qubits on an NMR quantum computer. For the one- and the two-bit \\ Deutsch problem, the qubits do not get entangled, and the NMR implementation is achieved without \\ using spin-spin interactions. It is for the three-bit case, that the manipuation of entangled states \\ becones essential. The interactions through scalar $I$-couplings in NMR spin systems have been \\ exploited to inplement entangling transformations required for the three bit DJ algorithn.
}

Keywords. Quantum computation; entanglement; nuclear magnetic resonance; $D$ J-algorithm.

PACS Nos 03.67.Lx; 76.70.-k

The utilization of the intrinsically quantum mechanical nature of the physical world to widen the scope of computational algorthms is one of the important discoveries of this decacle [1-3]. It was shown recently that quantum computers can perform certain conputational tasks nontrivially faster than classical computers [4-6]. NMR has been the nost successful technique to implement quantum algorithms till date $[7,8]$, even though the debate continues about its implications [9].

The Cleve version of the DJ algorithm [10], which requires $n+1$ qubits to solve the $n$-bit Deusch problem, has been implemented by several research groups using NMR [1 1- [4]. It has been shown recently that the n-bit Deutsch problem can be solved using $n$ qubits alone [15].

In this paper, we experimentally demonstrate that the $n$-bit. DJ algorithm does not require $n+1$ qubits for its implementation. By doing away with the extra qubit, the algorithm can be more easily accessed for a greater number of qubis. Furthermore, the one-bit and two-bit implementations of the modified version do not involve quantum entanglement $[15,16]$. In these cases, only the concept of coherent superposition is exploited, to prepare 'it parallel' an input state which is a superposition of all possible classical inputs, and the experiment has been perfomed without using spin-spin interactions. It is in the implementation for three or more qubits that quantum entanglement plays a vital role. It hence emerges from our results that the D, problem for one and wo input qubits is not 
in essence a quantum problem. The non-trivial guantum features in the algorithm show up only in the implementation for three or more qubits. The NMR implementation of the modified DJ algorithm for one and two qubits uses selective pulses that achieve rotations in the subspaces of individual spins. The non-trivial phase shifts essential for the threebit problem have been implemented using composite $z$-pulses. A judicious combination of selective if pulses and free evolution intervals (under the interaction Hamiltonian), has been employed to construct the required entangling transformations.

Consider an $n$-bit binary stritg $x$; a function $f$ can be defined on this $n$-bit domain space to a 1-bit range space, with the restriction that either the output is the sane for all inputs (the function is 'constanc') or the output is 0 for half the inputs and 1 for the other half (the function is 'balanced'). A.l the $2^{n}$ possible input strings are valid inputs for the function $(f(n)=\{0,1\})$. In quantum comptatation, these $n$-bit logical stings are in one-to-one cortespondence with the eigenstates of nucqubits, and one can hence label the logical string $x$ by the eigenstate $|x\rangle$. Classically, for an $n$-bit domain space, one needs to compute the function at least $2^{n-1}+1$ times in order to determine whether it is constant or balanced. The DJ algorithm achieves this on a quantum computer using only a single function call $[4,10]$.

The usual implementation of the D.J algorithm for $n$ bits requires $n+1$. qubits, the function $f$ being encoded through an $f$-dependent unitary transformation

$$
|x\rangle_{n-\text { bit }}|y\rangle_{1-\text { bit }} \stackrel{U_{S}}{\longrightarrow}|x\rangle_{n-\text { bit }}|y \oplus f(x)\rangle_{1 \text {-bit }},
$$

where $\rightarrow$ denotes addition modulo 2 . The implementation of the unitary transformation $U_{f}$, along with the Hadamard transformation, then suffices to distinguish the function as constant or balanced $[4,[0]$. A Hadanard transformation on one qubit mixes the eigenstates maximally,

$$
\begin{aligned}
& |0\rangle \stackrel{H}{\rightarrow} \frac{1}{\sqrt{2}}(|0\rangle+|1\rangle) \\
& |1\rangle \stackrel{H}{\rightarrow} \frac{1}{\sqrt{2}}(|0\rangle-|1\rangle)
\end{aligned} ; H=H^{-1}=\frac{1}{\sqrt{2}}\left(\begin{array}{rr}
1 & 1 \\
1 & -1
\end{array}\right) .
$$

The Hadamard transformation for $n$-qubits is the tensor product of the one-qubit transformation ( $H^{n}=H \otimes H \otimes H \otimes \cdots \otimes H$ ), its action on the $n_{\text {-qubit eigenstates being }}$

$$
H^{n}|x\rangle=\frac{1}{2^{n / 2}} \sum_{y=0}^{2^{n}-1}(-1)^{\oplus \sum_{j} n_{j} y_{j}}|y\rangle,
$$

where $x_{j}$ and $y_{j}$ are the $j$ th entries of the n-bit strings $a$ and $y$.

A modified scheme can be designed to solve the $n$-bit Deutsch problem, using $n$ qubits alone [15]. Here, for every function $f$ a unitary transformation is constructed, such that its action on the eigenstates of $n$-qubits is

$$
|x\rangle_{n-\text { biti }} \stackrel{U_{f}}{\longrightarrow}(-1)^{f(x)}|x\rangle_{n-\text { bit }} .
$$

It is instructive to explore the relationship between eqs (1) and (4) and to see how exactly we are able to get rid of the extra qubit for the function call required in the older scheme. The original motivation behind including the extra qubit for the function call in eq. (1) was to construct a reversibie(unitary) way of implementing otherwise irreversible function $f$. 
Quantum entanglement

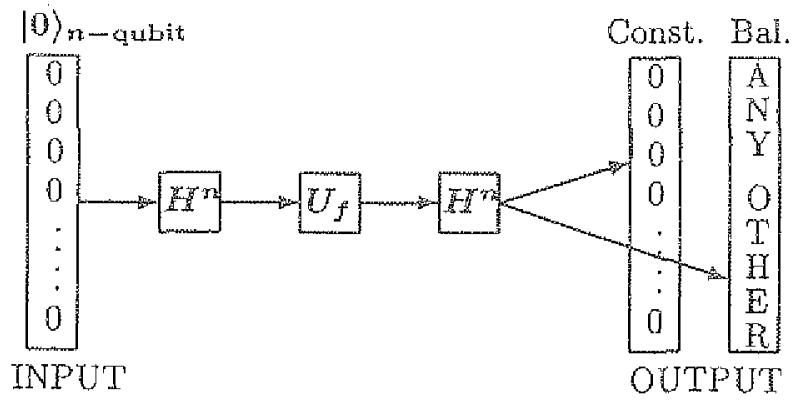

Figure 1. The block diagram for the modifed DJ algorithn.

However, it turns out that we can untarily implement $f$ without this extra gubit. The way the function is being implemented in eq. (4) is through phase shifts which is a purely quantum mechanical concept and has no classicat analogue. On the other hand, eq. (1) has a classical motivation and it indeed reduces to a classical implementation if applied to just the eigenstates of the qubits involved. This brings out the interesting fact that one should not always use classical ways of implementing logic even on eigenstates, and it might be useful to directy implement quantum schemes without botheting about corresponding classical analogues.

Consider $n$ qubits, all in the state $|0\rangle$; a Hadamard transformation $H^{n}$ converts this state to a linear superposition of all $2^{n}$ eigenstates with equal amplitudes and no phase differences. The unitary transformation $U_{f}$ (defined in eq. (4)) acting on this state, introduces an $f$-dependent phase factor in each eigenstate in the superposition. At this juncture, all information about $f$ is encoded in the quantum state of the qu qubis. A Hadamard transformation $H^{\prime 2}$ is once again applied in order to extract the function 's constant or balanced nature:

$$
\begin{aligned}
&|0\rangle \stackrel{r^{n}}{\rightarrow} \frac{1}{2^{n / 2}} \sum_{n=0}^{2^{n}-1}|x\rangle \stackrel{U_{f}}{\rightarrow} \frac{1}{2^{n / 2}} \sum_{n=0}^{2^{n}-1}(-1)^{f(x)}|x\rangle \stackrel{H^{n}}{\rightarrow} \\
& \frac{1}{2^{n}} \sum_{x=0} \sum_{y=0}^{2^{n}-1}(-1)^{2^{n}-1}(x)(-1)^{\oplus \sum_{j} x_{j} y_{j}|y\rangle .}
\end{aligned}
$$

The final expression for the output state in eq. (5) has an amplitude 1 for the state $|0\rangle_{n-b i t}$ for a constant function and an amplitude 0 for a balanced function.

The categorization of the function as constant or balanced through a single function call using $n$ qubits, is shown pictorially in figure 1.

The number of functions for the n-bit Deutsch problem is ${ }^{N} C_{N / 2}+2$ (where $N=2^{n}$ ). The experimental implementation of the modified DJ algorithm for $n$ bits requires the realization of the mintary transfomation corresponding to each of these functions, alongwits the mbit Hadamard transformation, on a physical system. We now proced towards the NMR implementation of the modiled DJ algotinn for one, wo and thee qubits, where the number of functions are 4,8 , and 72 respectively.

The pseado-Hadamard transtormation $[17]$ (practically equivalent to the Hadanard operator described in eq. (2)) achieved by a $\left(90^{\circ}\right)$, pulse on a spin, has been utilized in our 
Arvind, Kavita Dorai and Anil Kumar
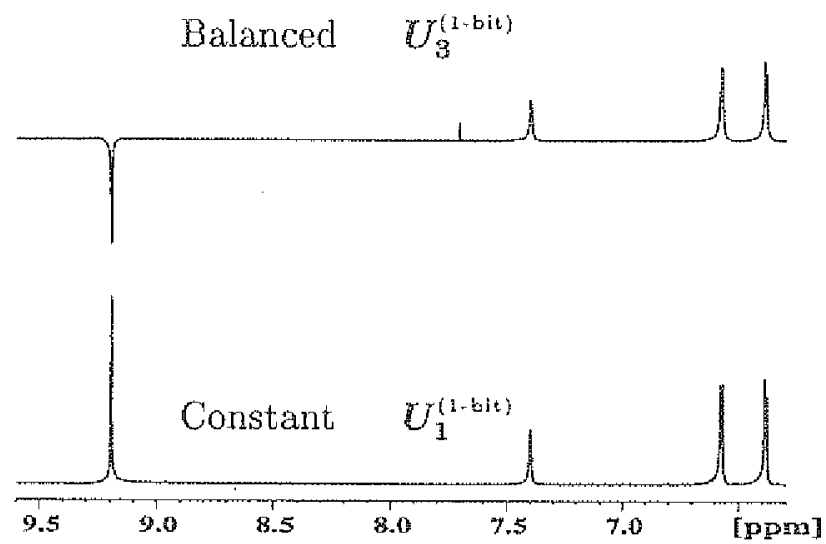

Figure 2. The modified DJ algorithm for one qubit implemented on 5-nitro-2-furaldehyde, the proton resonating at $9.2 \mathrm{ppm}$ being chosen as the computation qubit.

experiments. For the case of two and three qubits, the same has been achieved by a $\left(90^{\circ}\right)_{y}$ pulse applied non-selectively on all the spins.

The n-bit unitary transformations $U_{f}$ corresponding to the functions $f$, are diagonal in the eigenbasis and find a natural description in terms of the single-spin operators,

$$
I^{(j)}=\left(\begin{array}{ll}
1 & 0 \\
0 & 1
\end{array}\right) \quad ; \quad \sigma_{z}^{(j)}=\left(\begin{array}{cc}
1 & 0 \\
0 & -1
\end{array}\right),
$$

where $j$ labels the qubit involved. The action of $\vec{U}_{f}$ on an eigenstate (as described in eq. (4)), has been used to calculate the explict matrix forms of $U_{f}$, for every function $f$.

The operator representations of the four unitary transformations for the one-bit modified DJ algorithm are

$$
\begin{aligned}
& U_{1}^{(1-\mathrm{bit})}=I^{(1)} \quad \text { (const.), } \\
& U_{2}^{(1-\text { bit })}=-I^{(1)} \quad \text { (const.), } \\
& U_{3}^{(1-\text { bit })}=\sigma_{z}^{(1)} \quad \text { (bal.), } \\
& U_{i}^{\left(1-b i i^{\prime}\right)}=-\sigma_{2}^{(1)} \text { (bal.). }
\end{aligned}
$$

A psendo-Hadamatd operation achieved by a $\left(90^{\circ}\right)$ y pulse is applied on a thermal initial state, in order to create a coherent superposition prior to applying the desired unitary transformations $U_{f}$. The constant functions correspond to a 'do-nothing' operation, while the balaned functions ate achieved by a rotation by the angle $\pi$ about the $z$-axis of the spin, up to a global phase factot. These z-rotations have been implemented using composite-z pulses, whereby a rotation by an arbitrary angle $\theta$ about the $z$-axis, can be decomposed as a set of rotations about the $x$ and $y$ axes [18]:

$$
(\theta)_{z} \equiv(\pi / 2)_{w}(\theta)_{y}(\pi / 2)_{-n}
$$

Global phase changes are not detectable in NMR and are hence ignored. The spectum reflects the constant or balanced nature of the lunction implenented (igure 2). After the 
Quanim entanglement

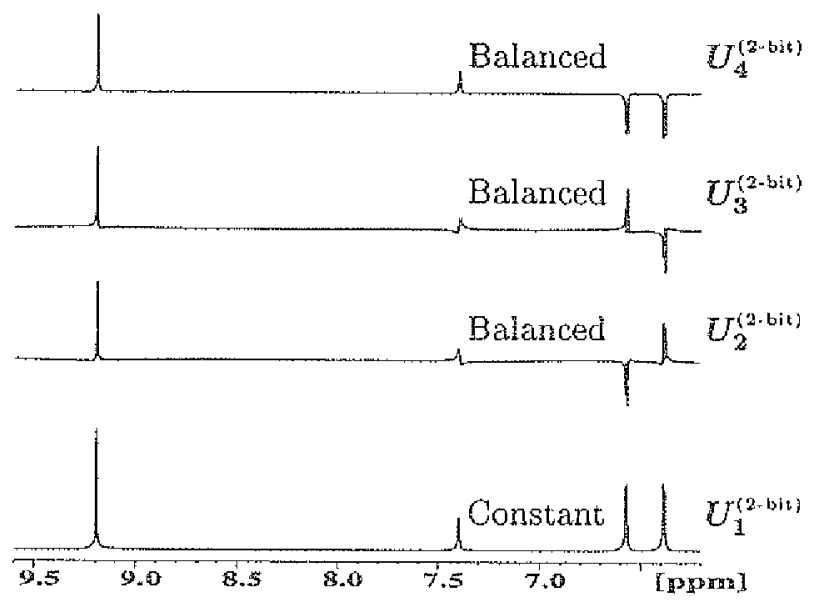

Figure 3. The modified DJ algorithm for two qubits, implemented on 5-nitro-2-furaldehyde with the two qubits resonating at 6.47 ppu and 6.29 ppin respectively.

implementation of a balanced function, the qubit is in a state out-of-phase with the rest of the spectrum. The modified DJ algorithm for one qubit demonstrates the power inherent in even a single bit of quantum information.

All the 8 unitary transformations corresponding to the functions for the two-qubit case are given in tems of $I^{(j)}$ and $\sigma_{z}^{(j)}$ as

$$
\begin{aligned}
& U_{1}^{(2-\mathrm{bit})}=I^{(1)} \otimes I^{(2)} \quad \text { (const.), } \\
& \left.U_{2}^{(2-\text { bit })}=\sigma_{z}^{(1)} \otimes I^{(2)} \quad \text { (bal. }\right)_{2} \\
& U_{3}^{(2-\text { bit })}=I^{(1)} \otimes \sigma_{z}^{(2)} \quad \text { (bal.), } \\
& U_{i}^{(2-\text { bit })}=\sigma_{z}^{(1)} \otimes \sigma_{z}^{(2)} \quad \text { (bal.), } \\
& U_{i}^{(2-\mathrm{bil})}=-U_{i-4}(i=5,6,7,8) \text {. }
\end{aligned}
$$

All these operators are direct products of single spin operators. They are thus incapable of generating entangled states and can be implemented by operations on individual spins. A pseudo-Hadamard transformation was performed on all the spins (initially in thermal equilibrium) prior to the execution of the desired $U_{i}$ transformations. The two constant functions correspond to the 'do-nothing' operation, experimentally. The NMR implemen-

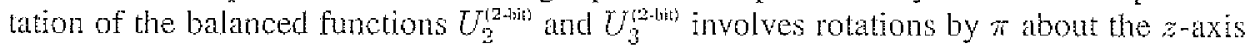
in the single-spin subspaces of spins 1 and 2 respectively, and have been achieved using composite- $z$ pulses (eq. (8)). The $U_{d}^{[2 \text { birt }}$ transformation too, does not require the scalar I spin interaction and is inplemented as successive $\pi$ rotations about the $z$-axes of spins $I$ and 2 respectively. The balanced functions are distinguished by one (or both) the spins being out-of-phase with the rest of the NMR spectum (figure 3). Only half the total number of functions have been shown in the one and two-qubit cases, as the others are merely negatives of these, and lead to the same spectral patterns (spectra not shown).

The three-qubit Dy algorithm affords the simplest example where quantum entanglenent plays a definitive role in the computation. We reiterate here, that a computation is truly 
guatum in chancter only when entanglement is present. The task here is to implement 72 unitary transformations, the explicit operator forms for 9 of which are

$$
\begin{aligned}
& U_{1}^{(3-b i t)}=I^{(1)} \otimes I^{(2)} \otimes I^{(3)} \text { (const.), } \\
& U_{2}^{(3-\mathrm{bit})}=\sigma_{z}^{\{1\}} \odot I^{(2)} \otimes I^{(3)} \quad \text { (bal) } \\
& U_{3}^{(3-b i t)}=J^{(1)} \otimes \Gamma^{(2)} \otimes \sigma_{z}^{(3)} \text { (bal.) } \\
& U_{4}^{(3-b i t)}=\sigma_{z}^{(1)} \otimes \sigma_{z}^{(2)} \otimes I^{(3)} \text { (bal.) } \\
& U_{5}^{(3-\text { bit })}=\sigma_{4}^{(1)} \otimes \sigma_{z}^{(2)} \otimes \sigma_{z}^{(3)} \text { (bal.), } \\
& O_{6}^{(3-\mathrm{bit})}=\frac{1}{2} \sigma_{z}^{(1)} \otimes\left(I^{(2)} \otimes I^{(3)}+\sigma_{z}^{(2)} \otimes I^{(3)}\right. \\
& \left.+I^{(2)} \otimes \sigma_{z}^{(3)}-\sigma_{z}^{(2)} \otimes \sigma_{z}^{(3)}\right) \text { (bal) } \\
& U_{7}^{(3-\mathrm{bit})}=\frac{1}{2} \sigma_{z}^{(2)} \otimes\left(I^{(1)} \otimes I^{(3)}+\sigma_{z}^{(1)} \otimes I^{(3)}\right. \\
& \left.+I^{(1)} \otimes \sigma_{z}^{(3)}-\sigma_{z}^{(1)} \oslash \sigma_{z}^{(3)}\right) \text { (bal.) } \\
& U_{g}^{(3-\mathrm{pib})}=\frac{1}{2} \sigma_{z}^{(3)} \otimes\left(I^{(1)} \otimes I^{(2)}+\sigma_{z}^{(1)} \otimes I^{(2)}\right. \\
& +I^{(1)} \otimes \sigma_{\tilde{z}}^{(2)}-\sigma_{\dot{z}}^{(\mathrm{E})} \otimes \sigma_{z}^{(2)} \text { ) (bal.), } \\
& U_{9}^{(3-\text { bit })}=\frac{1}{2}\left(\sigma_{z}^{(1)} \otimes I^{(2)} \otimes I^{(3)}+I^{(1)} \otimes I^{(2)} \otimes \sigma_{z}^{(3)}\right. \\
& -\sigma_{z}^{(1)} \otimes \sigma_{z}^{(2)} \otimes I^{(3)}+I^{(1)} \otimes \sigma_{z}^{(2)} \otimes \sigma_{z}^{(9)} \text { ) (bal.). }
\end{aligned}
$$

The operators $U_{1}^{(3-\mathrm{bir})} U_{i^{3}}^{(3 i)}$ can be decomposed as direct products of single-spin operators and are thbus non-entangling transformations. The operators $U_{6}^{(i-b i l)}-U_{9}^{(3-\sin )}$ cannot be decomposed as clirect products of single-spin operators and are hence capable of generating entangled states from non-entangled ones. The operators $U_{0}^{(3-\text { thit }}$, $U_{7}^{\{3 \text { bit }}$, and $U_{3}^{(3-\text { bil }}$, are entangling in different two-spin subspaces and can be factored as direct products of a single-spin operator and a two-particle entangling transformation. They can thus generate states in which two qubits are entangled, with the third qubit remaining non-entangled with either of them. On the other hand, the transformation $U_{9}^{\text {tath }}$ does not allow any such simm plifications, It is maximally entangling and leads to states that are three-cubit entangled. These 9 functions are thus divided into three categories namely, non-entangling, two-qubit entangling, and maximally (three-qubit) entangling. The remaining functions are similar in form, and can be classed into one or the other of these categories.

The result of experimentally applying the non-entangling transformations $U_{1}^{\text {(3-lil) }}-U_{5}^{\text {(3-tir) }}$, after a pseudo-Hadamad transformation on a thermal equilibrum state, is shown in figure 4. The constant function $U_{1}^{(3-\text { lir }}$ is the unit operator, and comesponds to the 'no pulse' or the 'do-nothing' operation. The balanced functions $U_{2}^{\text {thit }}$ and $U_{3}^{\text {(3) }}$ correspond to a rotation by the angle $\pi$ about the $z$-axis of the first and the thro spins respectively, without perabing the other spins. This has been acheved by a spin-selective $[\pi]$, puise in each case, using composite $z$-pulses (eq. (8)). The spectrum is categorized by the spin in guestion being out-of-phase with the rest of the spectrum. The transformation $U_{*}^{(3-h i n}$ has

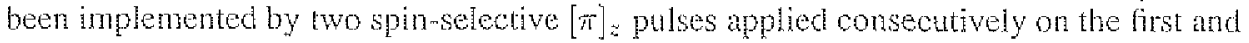
the second spins respectively, and leads to a spectrum with both these spins being ont-ofphase with the third. The non-entangling balanced function $U_{5}^{\text {(3-list }}$, has been implemented by successive spin-selective $[\pi]_{z}$ pulses on all the thee spins.

The wo-qubit entangling transformation $V_{6}$, is achieved by the pulse sequence

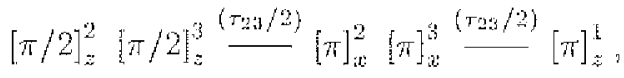




\section{Qiantum entanglement}

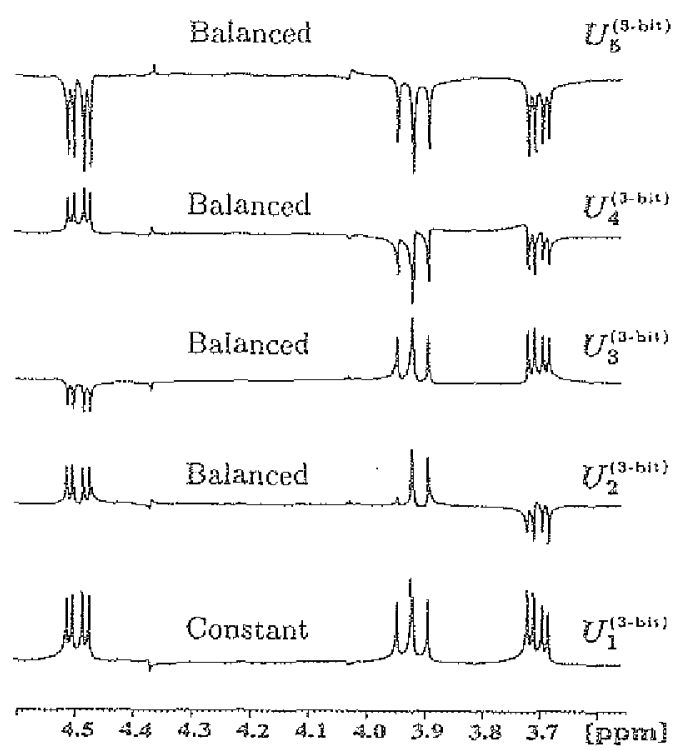

Tigure 4 . The refined DJ algorithm for three qubits, implemented on 2,3-dibromopropionic acid. The functions siown are all non-entangling in nature.

where $\tau_{23}=1 / J_{23}$ and 1,2 and 3 are qubit labels. The operators $U_{7}^{(3 .-b i l\}}$ and $U_{8}^{(i 3-3 i l)}$ correspond to cyclic pernutations of the qubits. The spin-selective $\pi$ pulses in the middle of the free evolution period $\tau_{23}$ refocus the chenical shift evolution. The pulse sequence (applied after a pseudo-Hadamard transformation on all thee gubits in a themal initial state) resuls in a density matrix with the product operator form $-I_{x}^{1}+2 I_{x}^{2} I_{z}^{3}+2 I_{z}^{2} T_{x}^{3}$, leading to a spectrum with the multiplet of the first qubit inverted, and an antiphase doublet of doublet patten for the other two qubits (figure 5). The three-qubit entangling function $U_{0}^{[3.6 \mathrm{kin})}$ is implemented by the pulse sequence

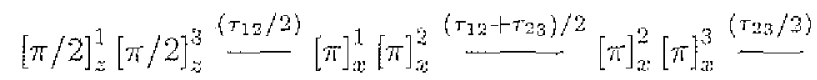

leading to an antiphase spectral pattern for all three qubits, corresponding to the product operators $2 I_{z}^{1} I_{z}^{2}+2 I_{z}^{2} I_{z}^{3}+4 I_{z}^{1} I_{z}^{3} I_{z}^{3}$ (figure 5 ). The spectra in figure 5 suffer from phase distortions arising from the inaccurate relocusing of the chemical shifts durng the $\tau$ perods, and $J$-evolution during long spin-selective composite-z puises $\left([\pi]_{z}^{i} \equiv[\pi / 2]_{z}^{i}[\pi]_{b /}^{i}[\pi / 2]_{-z}^{i}=\right.$ 42 misec).

This implenentation of the DJ algorithn does not require the initial preparation of the spins in a pseudo-pure state, since the thermal equilibrum state serves equally well as a good initial state. The observable spectrat result is the same in both cases, though beginning with a psendo-pure state creales sone (undetectable) multiple-quantum coherences. Further, it is interesting to note that the application of an entangling transfomation to an zinentangled state does not al ways lead to an entangled state. An particular, for the three bit DJ algorithm implemented on thermal initial states, the final output state (carying information about the function's constant or balanced nature), is unentangled, even though some of the unitary transtormations requited are entangling in natue. 
Anind, Kavita Dorai and Anil Kumar

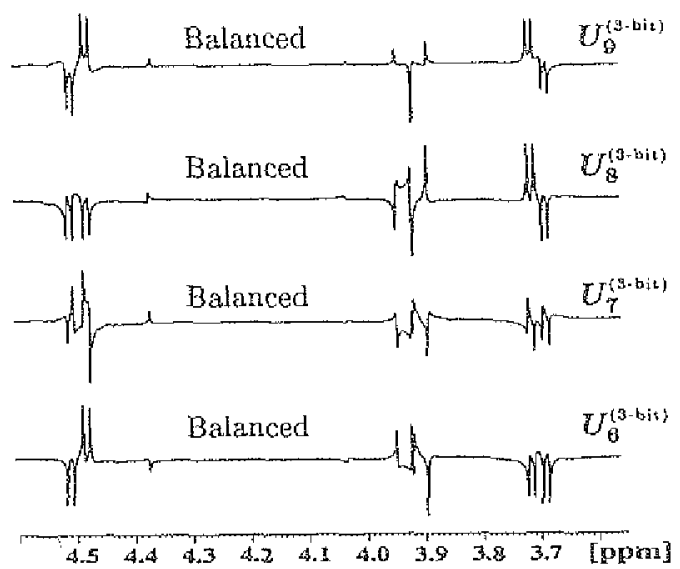

Frigure 5. Entangling balanced functions implemented on the three-qubit system of 2,3-dibromopropionic acid.

The final pseudo-Hadamard transformation to extract the constant or balanced nature of the function (figure 1 ) is cancelled by the $(90)^{\circ}$ read-out pulse usually used in NMR experiments, and the computation essentially culminates in the application of the desired $U_{f}$ function after the first pseudo-Hadamard transformation.

A nodification to the usual Df agorithm enabled an n-bit implementation using $n$ qubits. The required unitary transtormations were tailored to eliminate the need for the extra qubit, and the modified DJ algorithm was tested experimentally for one, two and three qubits. While the one and two qubit cases use non-entangling unitary transformations, it was noted that for three (or more) qubits, multi-particle entangling tansformations are essential. The NMR implementation of such entangling transformations requires the presence and manipulation of spin-spin inferactions [19].

\section{Acknowledgenent}

The use of the AMX 400 spectrometer at SIF, ITSc, Bangalore, funded by DST, New Delhi, is gratefully acknowledged.

\section{References}

[1] DP Divincenzo, Science 270, 255(1995)

[2] S Lloyd, Sci. Am. 273, 44 (1995)

[3] I L Chuang, R Laftamme, P W Shor and W H Zurek, Science 270, 1633 (1995)

[4] D Deutsch and R. Jozsa, Proc. R. Soc. London A439, 553 (1992)

[5] P W Shot, SIAM J. Comput. 26, 1484 (1997)

[6] L K Grover, Phys. Rev. Lett. 79, 325 (1997)

[7] D G Coly, A F Fahmy and T F Havel Froc. Natl. Acal. Sci. USA 94, 1634 (1997)

[8] N Garshenfeld and I L Chumg, Science 275, 350 (1997) 
[9] S L Bramstein et al, Pfyss. Rev. Lett. 83, 1054 (1999)

[10] R Cleve, A Ekert, C Macchiavello and M Mosca, Proc. R. Soc. London A454, 339 (1998)

[11] I L Chung, I. M K Vandersyper, X Zbou, D W Leung and S Lloyd, Nature 393, 143 (1998)

[12] J A Jones and M Mosca, J. Chem. Phys. 109, 1648 (1998)

[13] N Linden, H Barjat and R Freeman, Chem. Phys. Lett. 296, 61 (1998)

[14] Kavita Dorai, Arvind and Anil Kumar, Phys. Rev. A61, 042306/1-7 (2000)

[15] D Collins, K W Kim and W C Holton, Phys. Rex A58, R1633 (1998)

[161 A Ekert and P L Knight, Am. J. Phys. 63, 415 (1995)

[17] J A Jones, R H Hansen and M Mosca, J. Magn. Reson. 135, 353 (1998)

[18] R R Enst, G Bodenhausen and A Wokaun, Principles of miclear magnetic resonance in one and two dimensions (Oxford University Press, Oxford, 1994)

[19] Arvind, Kavita Dorai and Anil Kumar, LANL e-print quant-ph/9909067 